\title{
Long-term AGILE monitoring of the puzzling gamma-ray source 3EG J1835+5918
}

A. Bulgarelli ${ }^{1}$, M. Tavani ${ }^{2,5}$, P. Caraveo ${ }^{4}$, A. W. Chen ${ }^{3,4}$, F. Gianotti ${ }^{1}$, M. Trifoglio ${ }^{1}$, M. Marelli $^{4}$, A. Argan $^{2}$, G. Barbiellini ${ }^{6}$, F. Boffelli ${ }^{9,15}$, P. W. Cattaneo ${ }^{9}$, V. Cocco $^{2}$, E. Costa ${ }^{1}$, F. D’ Ammando ${ }^{2,5}$, E. Del Monte ${ }^{2}$, G. De Paris ${ }^{2}$, G. Di Cocco ${ }^{1}$, I. Donnarumma ${ }^{2}$, Y. Evangelista ${ }^{2}$, M. Feroci ${ }^{2}$, M. Fiorini ${ }^{4}$, T. Froysland ${ }^{2,5}$, F. Fuschino ${ }^{3}$, M. Galli ${ }^{7}$,

A. Giuliani ${ }^{4}$, C. Labanti ${ }^{1}$, I. Lapshov ${ }^{2}$, F. Lazzarotto ${ }^{2}$, P. Lipari ${ }^{8}$, F. Longo ${ }^{6}$, M. Marisaldi ${ }^{1}$, S. Mereghetti $^{4}$,

A. Morselli ${ }^{10}$, L. Pacciani ${ }^{2}$, A. Pellizzoni ${ }^{4}$, F. Perotti ${ }^{4}$, G. Piano ${ }^{2,5}$, P. Picozza ${ }^{5,10}$, M. Prest ${ }^{11}$, G. Pucella ${ }^{2}$,

M. Rapisarda ${ }^{12}$, A. Rappoldi ${ }^{9}$, P. Soffitta ${ }^{2}$, A. Trois ${ }^{2}$, E. Vallazza ${ }^{6}$, S. Vercellone ${ }^{4}$, V. Vittorini ${ }^{2,3}$, A. Zambra $^{4}$, D. Zanello ${ }^{8}$, P. Giommi ${ }^{13}$, C. Pittori ${ }^{13}$, F. Verrecchia ${ }^{13}$, P. Santolamazza ${ }^{13}$, D. Gasparrini ${ }^{13}$, S. Cutini ${ }^{13}$, S. Colafrancesco ${ }^{13}$, and L. Salotti ${ }^{11}$

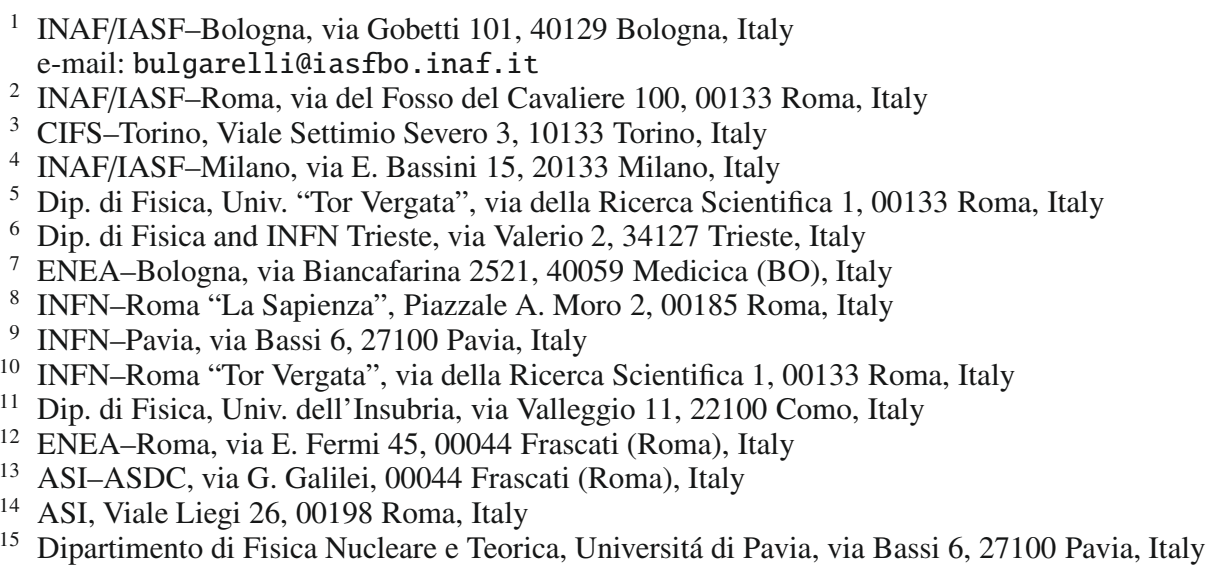

Received 30 July 2008 / Accepted 13 August 2008

\section{ABSTRACT}

Context. We present the AGILE gamma-ray observations of the field containing the puzzling gamma-ray source 3EG J1835+5918. This source is one of the most remarkable unidentified EGRET sources.

Aims. An unprecedentedly long AGILE monitoring of this source yields important information on the positional error box, flux evolution, and spectrum.

Methods. 3EG J1835+5918 has been in the AGILE field of view several times in 2007 and 2008 for a total observing time of 138 days from 2007 Sep. 04 to 2008 June 30 encompassing several weeks of continuous coverage.

Results. With an exposure time approximately twice that of EGRET, AGILE confirms the existence of a prominent gamma-ray source (AGL J1836+5926) at a position consistent with that of EGRET, although with a remarkably lower average flux value for photon energies greater than $100 \mathrm{MeV}$. A 5-day bin temporal analysis of the whole data set of AGL J1836+5926 shows some evidence for variability of the gamma-ray flux. The source spectrum between $100 \mathrm{MeV}$ and $1 \mathrm{GeV}$ can be fitted with a power law with photon index in the range 1.6-1.7, fully consistent with the EGRET value.

Conclusions. The faint X-ray source RX J1836.2+5925 that has been proposed as a possible counterpart of 3EG J1835+5918 is well within the AGILE error box. Future continuous monitoring (both by AGILE and GLAST) is needed to confirm the gamma-ray flux variability and to unveil the source origin, a subject that is currently being pursued through a multiwavelength search for counterparts.

Key words. gamma rays: observations

\section{Introduction}

The gamma-ray source 3EG J1835+5918 (also known as GEV $\mathrm{J} 1835+5921$ and GRO J1837+59) is one of the most puzzling high-energy sources in the sky, and it has been the subject of considerable interest since its discovery by EGRET (Nolan et al. 1994). This source was catalogued among the brightest highlatitude unidentified sources with an average gamma-ray flux above $100 \mathrm{MeV}$ of $\Phi_{\mathrm{EGRET}}=(60 \pm 4) \times 10^{-8}$ photons $\mathrm{cm}^{-2} \mathrm{~s}^{-1}$, and a hard gamma-ray spectrum of photon index $\gamma=1.69 \pm 0.07$ (Hartman et al. 1999). Owing to the spectral hardness (similar to that of the Vela pulsar), the source flux near $1 \mathrm{GeV}$ is expected to be about half of the Crab value. The EGRET error box is well-determined, resulting in an error radius of $8^{\prime}$ at the $95 \%$ confidence level. 
Table 1. AGILE observations of 3EG J1835+5918.

\begin{tabular}{|c|c|c|c|c|c|c|c|c|c|}
\hline Viewing period & $l$ & $b$ & Start (UTC) & End (UTC) & Off-axis angle & $F_{\gamma}$ & $\Delta F_{\gamma}$ & Counts & $\sqrt{(T S)}$ \\
\hline OB2300 & 134.882 & 11.822 & $2007-09-04$ 12:00 & $2007-09-12$ 12:00 & 50 & $<40$ & & $<13$ & 1.8 \\
\hline $\mathrm{OB} 4610+\mathrm{OB} 4630$ & 143.365 & 41.588 & $2007-10-24$ 08:00 & $2007-11-01$ 12:00 & 50 & 81 & 18 & $37 \pm 10$ & 4.4 \\
\hline OB4800 & 69.594 & 4.623 & $2007-11-02 \quad 12: 00$ & 2007-12-01 12:00 & 27 & 40 & 6 & $99 \pm 16$ & 7.7 \\
\hline OB4900 & 88.815 & 9.928 & $2007-12-01$ 12:00 & 2007-12-05 09:00 & 15 & 39 & 14 & $18 \pm 5$ & 3.8 \\
\hline OB $4910+$ OB 4920 & 85.119 & -9.416 & 2007-12-05 09:00 & $2007-12-16$ 12:00 & 38 & 48 & 12 & $44 \pm 10$ & 5.2 \\
\hline OB5210 & 77.309 & 40.628 & 2008-02-09 09:00 & $2008-02-12 \quad 12: 00$ & 20 & 60 & 19 & $21 \pm 7$ & 4.2 \\
\hline OB5600 & 53.039 & 6.474 & 2008-04-10 12:00 & 2008-04-30 12:00 & 40 & 40 & 10 & $57 \pm 13$ & 5.0 \\
\hline OB5700 & 104.852 & 35.439 & $2008-04-30 \quad 12: 00$ & 2008-05-10 12:00 & 30 & 49 & 11 & $49 \pm 10$ & 6.2 \\
\hline OB5800 & 74.05 & 0.273 & $2008-05-10 \quad 12: 00$ & 2008-06-09 12:00 & 30 & 50 & 9 & $129 \pm 18$ & 9.6 \\
\hline OB5820 & 93.6 & -1.16 & $2008-06-15$ 12:00 & 2008-06-30 12:00 & 30 & 25 & 8 & $37 \pm 11$ & 4.2 \\
\hline
\end{tabular}

The source 3EG J1835+5918 is located $25^{\circ}$ off the Galactic plane and therefore is not significantly affected by the Galactic diffuse gamma-ray emission. EGRET pointed at this high-galactic latitude region several times (Hartman et al. 1999). The resulting position, gamma-ray lightcurve, variability, and spectrum have been discussed in several papers: (McLaughlin et al. 1996; Nolan et al. 1996; Reimer et al. 2001). The analysis of the whole EGRET dataset produces a gamma-ray flux database consistent with being constant (Hartman et al. 1999; Reimer et al. 2001). However, it is important to notice that a claim for variability of 3EG J1835+5918 was indicated by Nolan et al. (1996).

The relatively strong gamma-ray flux, the hard spectrum, and the lack of an obvious blazar counterpart in the EGRET error box of 3EG J1835+5918 spurred considerable interest from several observing groups. After extensive X-rays, radio, and optical coverage of the EGRET error box (Mirabal et al. 2000; Reimer et al. 2001; Totani et al. 2002; Halpern et al. 2002) the search for possible counterparts singled out RX J1836.2+5925, a relatively faint soft X-ray source with no radio or optical emission: a set of characteristics similar to those of middle-aged radio-quiet neutron stars (Caraveo et al. 1996). Thus, on the basis of the gamma-ray properties of 3EG J1835+5918, coupled with those of RX J1836.2+5925, the sources was named the next Geminga by (Halpern et al. 2002) owing to its Geminga-like phenomenology (Bignami \& Caraveo 1996). A search for X-ray pulsation, with period values ranging from $1 \mathrm{~ms}$ to $10 \mathrm{~s}$, yielded a $35 \%$ upper limit to the source-pulsed fraction (Halpern et al. 2007). In view of the much lower pulsed fractions measured for all known middle-aged neutron stars, such a result did not weaken this tentative identification.

Taking advantage of the AGILE unprecedented exposure gathered during the science verification phase and early pointings of the Cycle-1 program, in this Letter we are now able to assess the source characteristics anew. Section 2 describes the AGILE-GRID observations and main results, while their implications are discussed in Sect. 3.

\section{The AGILE GRID observations of 3EG J1835+5918}

The AGILE istrument (Tavani et al. 2008) is composed of three detectors: a Tungsten-Silicon Tracker (ST) (Barbiellini et al. 2002; Prest et al. 2003), with a large field of view ( 2.5 sr), optimal time resolution and angular resolution, and good sensitivity; a Silicon-based X-ray detector, Super-AGILE (SA) (Feroci et al. 2007) for imaging in the energy range $18 \mathrm{keV}-60 \mathrm{keV}$ and a CsI(Tl) Mini-Calorimeter (MCAL) (Labanti et al. 2006) that detects gamma-rays or particle energy depositions between $300 \mathrm{keV}$ and $100 \mathrm{MeV}$. The ST and MCAL form the AGILE Gamma-Ray Imaging Detector (GRID) for observations in the gamma-ray energy range $30 \mathrm{MeV}-50 \mathrm{GeV}$. The instrument is completed by an anti-coincidence (AC) system (Perotti et al. 2006), made with plastic scintillator layers, for the rejection of charged particles, and by an efficient trigger logic for gamma-ray and X-ray data acquisition (Tavani et al. 2008).

Data analysis was performed with the BUILD 15 of the AGILE Standard Pipeline, publicly available at the ASI Data Center web site (http://agile.asdc.asi.it/). The data were produced starting from the Level-1 data. The events collected during the passage in the South-Atlantic Anomaly and the Earth albedo background were consistently rejected. The GRID event direction were reconstructed by a Kalman filter technique. To reduce the particle background contamination, we selected with the GRID filter FT3ab_2 only events flagged as confirmed $\gamma$-ray events ( $G$ class events, corresponding to a sensitive area of $\sim 300 \mathrm{~cm}^{2}$ at $\left.100 \mathrm{MeV}\right)$. All the fluxes reported in this Letter have been consistently checked and scaled with their corresponding errors using the ratio $R=\frac{\Phi(\text { Vela })_{\text {EGRET }}}{\Phi\left(\text { Vela }_{A G I E}\right.}$ computed for similar offaxis angles, with $\Phi$ (Vela) the gamma-ray flux above $100 \mathrm{MeV}$ reported by EGRET and measured by AGILE. Scaling our fluxes measured for 3EG J1835+5918 to the Vela PSR measurements by AGILE and EGRET also takes systematic effects into account (estimated to be of the order of the $10 \%$ of the fluxes reported here).

All AGILE observations of the field containing the 3EG J1835+5918 are listed in Table 1 . The columns report the viewing periods name (as reported in the ASI Data Center web site), the galactic coordinates of the centre of the field of view, the start and end of the observation in UTC, the average source off-axis angle, the period-averaged flux $F_{\gamma}(E>100 \mathrm{MeV})$ in photons $\mathrm{cm}^{-2} \mathrm{~s}^{-1}$ (if $\sqrt{T S}<3$ a $2-\sigma$ upper limit is reported), the $1-\sigma$ statistical and systematic uncertainty of the flux, the number of counts, and in the last column the statistical significance of the likelihood ratio test.

AGILE counts, exposure, and galactic background maps were generated with a bin size of $0.25^{\circ} \times 0.25^{\circ}$ for $E>$ $100 \mathrm{MeV}$ to compute the source flux and its evolution, while maps with a bin size of $0.1^{\circ} \times 0.1^{\circ}$ were used to optimize the source position using a likelihood analysis method (Mattox et al. 1996a). Our analysis was performed over a region of $10^{\circ}$ radius.

\subsection{The gamma-ray source positioning}

To determine the most likely location of the gamma-ray source, we used only the best viewing periods, i.e. those in which the 


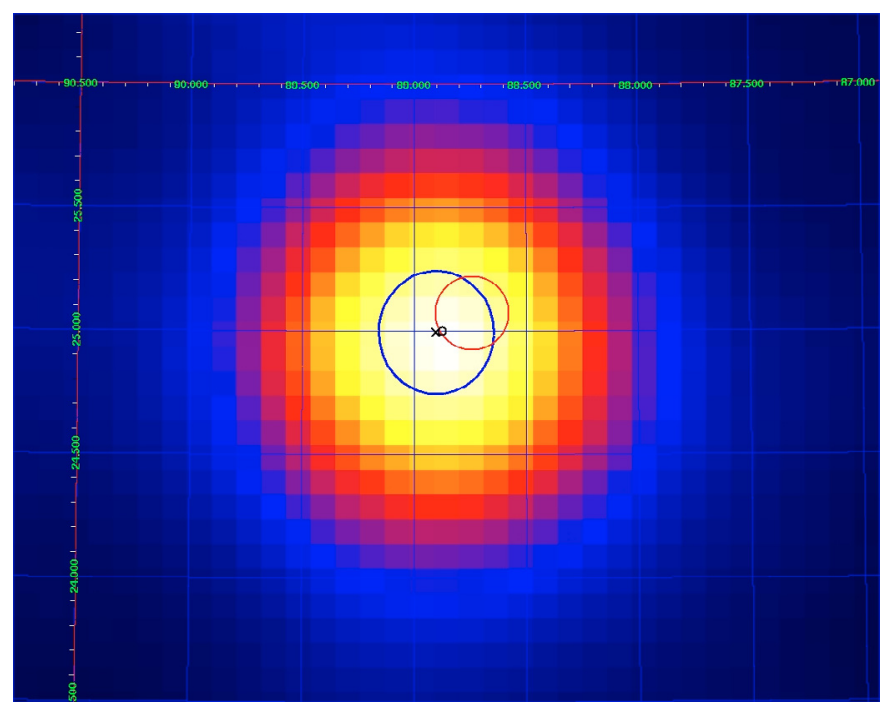

Fig. 1. Gaussian-smoothed counts map with squared scale of AGL J1836+5926 with photons of $E>100 \mathrm{MeV}$. The red curve is the EGRET 95\% error ellipse, the blue curve is the AGILE 95\% statistical plus systematic error ellipse, the cross is the AGILE 95\% error ellipse barycentre, and the black circle is the position of the X-ray source RX J1836.2+5925.

source was within $50^{\circ}$ from the instrument pointing direction. For $E>100 \mathrm{MeV}$, the best position computed by the likelihood analysis is $l=88.90^{\circ} b=24.99^{\circ}$, corresponding to $\alpha=18^{\circ} 36^{\prime} 20^{\prime} \cdot 11, \delta=59^{\circ} 26^{\prime} 42^{\prime \prime} \cdot 3$. We name this source AGL J1836+5926. Figure 1 shows a Gaussian-smoothed counts map of the source with our $95 \%$ statistical and systematic error ellipse with semiaxis $a=0.265^{\circ}$ and $b=0.200^{\circ}$. To conservatively take both systematic and statistical effects into account, we obtain a $95 \%$ confidence level radius by linearly adding a systematic error of $0.1^{\circ}$ to the $95 \%$ statistical error ellipse.

The AGILE 95\% error ellipse is consistent with the error box of 3EG J1835+5918 (Mattox et al. 2001), and the distance between AGILE and EGRET centroids is $\sim 10.2^{\prime}$. The distance between the position of the X-ray source RX J1836.2+5925 and the AGILE 95\% maximum likelihood contour level barycentre is $\sim 1.4^{\prime}$. The source detection significance, as derived from a maximum likelihood analysis, is $16.7-\sigma$. A total of 499 photons with $E>100 \mathrm{MeV}$ have been collected. The source flux, averaged over the whole AGILE dataset, is $\Phi_{\text {AGILE }}=(38.7 \pm 3.0) \times$ $10^{-8}$ photons $\mathrm{cm}^{-2} \mathrm{~s}^{-1}$, which turns out to be approximately $60 \%$ less than the value reported in the 3rd EGRET Catalogue.

\subsection{The gamma-ray spectrum}

Our standard GRID spectral analysis was performed using 3 energy bins, namely 100-200 MeV, 200-400 MeV, 400-1000 MeV. Photons below $100 \mathrm{MeV}$ and above $1 \mathrm{GeV}$ have not been used in the analysis reported here. The analysis was obtained by summing the observation periods OB5600, OB5700, and OB5800 (see Table 1). A power-law model fit to the data yields a photon index $\gamma=1.65 \pm 0.22$.

\subsection{The gamma-ray lightcurve and variability analysis}

The search for the variability of the gamma-ray emission of 3EG J1835+5918 produced somewhat inconclusive results. Using the first EGRET viewing periods, McLaughlin et al. (1996) and Nolan et al. (1996) classified 3EG J1835+5918 as a possibly variable source. On the other hand, using the whole EGRET data set Reimer et al. (2001) and Nolan et al. (2003) did not confirm the previous hint of variability. (We note that none of the previous EGRET variability studies used the same 5-day intervals adopted in this Letter.)

To study the source temporal behaviour, each AGILE viewing period was first analysed to determine the parameters of the Galactic diffuse radiation model and of the isotropic diffuse intensity level. The source flux density was then estimated independently for each 5-day temporal bin with the gammaray model parameters fixed at the values obtained in the first step. Figure 2 shows the AGL J1836+5926 gamma-ray flux lightcurve during the period September 2007 to June 2008 for photons with $E>100 \mathrm{MeV}$.

We fit the AGILE GRID fluxes to a constant value (the weighted mean of the average flux values), and computed the $V$ variability coefficient for the AGILE observations (McLaughlin et al. 1996). The 2- $\sigma$ upper limits (UL) were properly treated, assigning a value and a $\sigma$ equal to $U L / 2$ (Torres et al. 2001a). A value of $V>1$ is an indication of variability within the observing period. The AGILE observation block durations of Table 1 span a non-homogeneous range of timescales from 1 week to more than a month. To study the flux variability of AGL J1836+5926 on a timescale of a few days, we chose a temporal bin size of 5 days as a good compromise between statistics and variability characteristics. Using a 5-day bin size, we obtain $\chi^{2}=38.34$ for 24 degrees of freedom and a value of $V=1.495$. We can exclude the flux being constant at the $96.80 \%$ level. For a temporal bin of 4 days we obtain $V \simeq 1$. For temporal bins of 3 days or shorter, there are too many upper limits during the whole exposure time. For temporal bins of more than 5 days, we obtain $V<1$, as expected for averaging a signal potentially variable on a timescale of a few days.

We note that, during the long uninterrupted coverage obtained in Nov.-Dec. 2007 and in April-June 2008, the gammaray source AGL J1836+5926 was not detectable (failing to reach the 3- $\sigma$ threshold) for about half of the observing time. We also determined hard X-ray upper limits for AGL J1836+5926 in the energy range $18-60 \mathrm{keV}$ from an analysis of Super-AGILE data. A 3-sigma upper limit of $10 \mathrm{mCrab}$ was obtained for the OB5700.

\subsection{EGRET vs. AGILE}

After one year of operations, AGILE has doubled the EGRET exposure time of 3EG J1835+5918, confirming the source position and spectral shape and showing evidence of variability. A comparison of the AGILE and EGRET observations of 3EG J1835+5918 is reported in Table 2. With a few exceptions, the EGRET pointings were generally one-week long (Reimer et al. 2001), and they were not optimally suited to tracing an emission variable on week to month long timescales. On the other hand, AGL J1836+5926 has been continuously in the AGILE field of view for more than two months in April-June 2008, in addition to several weeks scattered between September 2007 and December 2007. It is too early to say whether the apparent variations are erratic or follow some kind of cyclic pattern. Longer, continuous exposures (both with AGILE and GLAST) are needed to clarify this crucial point.

\section{Discussion and conclusions}

The AGILE observations and monitoring of AGL J1836+5926 adds relevant information about this puzzling source. Postponing 


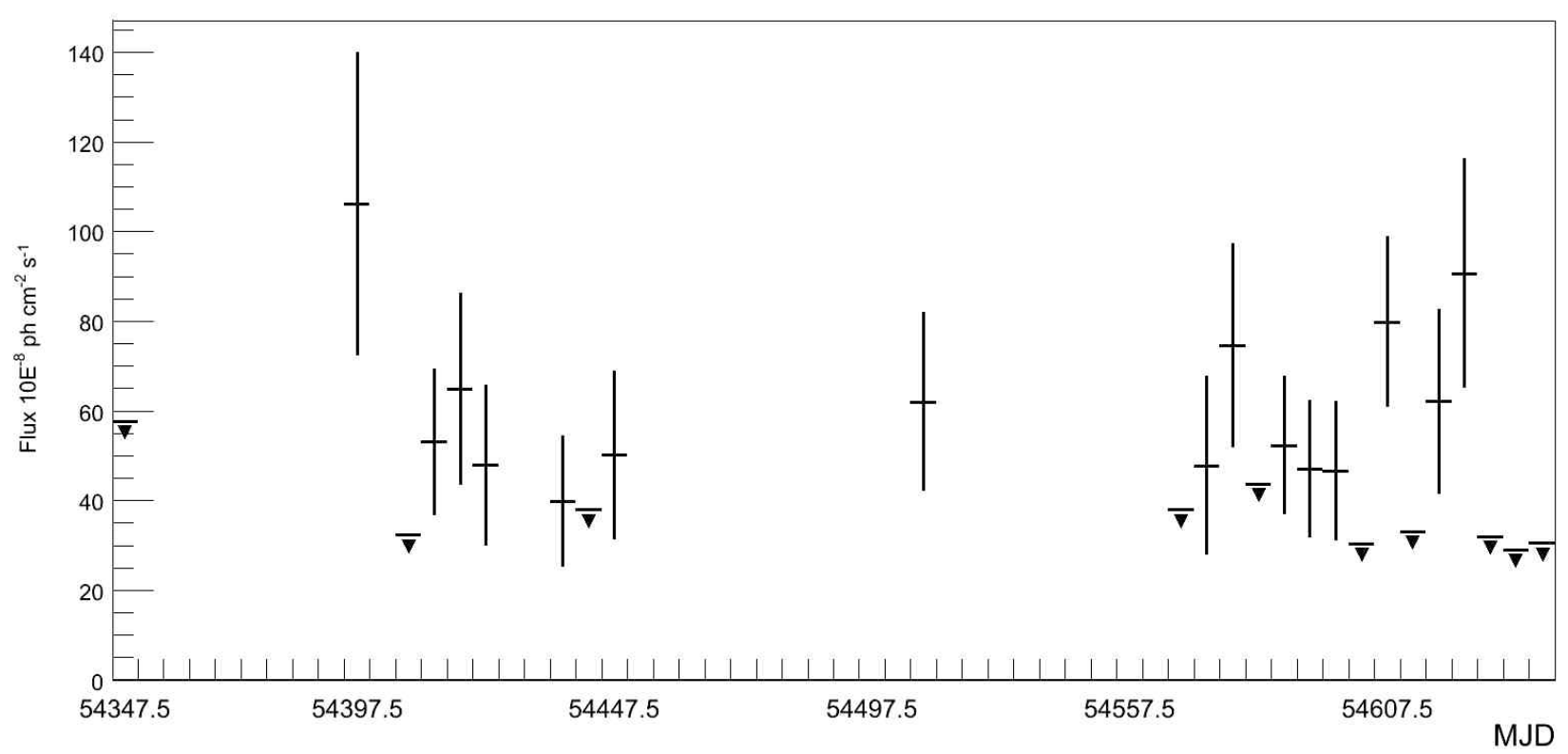

Fig. 2. AGILE gamma-ray light-curve of AGL J1836+5926 for photons above $100 \mathrm{MeV}$ with a 5-day temporal bin.

Table 2. Comparison of total exposure and number of photons gathered by EGRET and AGILE from 3EG J1835+5918 for $E>100 \mathrm{MeV}$.

\begin{tabular}{lll}
\hline \hline & $\mathrm{cm}^{2} \mathrm{~s} \mathrm{sr} / 0.5^{\circ}$ bin & Photons $E>100 \mathrm{MeV}$ \\
\hline EGRET & 52900 & 452 \\
AGILE & 118325 & 499 \\
\hline
\end{tabular}

an account of our multifrequency observations of the region to a forthcoming paper, we briefly outline here a few important points regarding the search for a counterpart. We confirm a point already noticed by other authors, i.e., the absence of a blazar or any other relatively bright radio sources in the field containing the AGILE (and EGRET) error box. The analysis of Mattox et al. (2001) shows a $100 \mathrm{mJy}$ source (B1834+5904) positioned at the $99.5 \%$ probability contour (12!7 from the EGRET centroid position and 21:8 from the AGILE centroid position). By using the NED, SIMBAD, and the Massaro et al. (2007) catalogues, we confirm the absence of a radio-loud blazar in the revised AGILE error box.

Outside this error box, we notice the existence of RGB J1841+591, a BL Lac type object positioned at 43.5 from the AGILE centroid position. Even though occasional contributions from this object cannot be excluded in our analysis of AGILE, we emphasise that the AGILE integrated flux positioning at the $95 \%$ contour level is clearly not consistent with any substantial contribution from RGB J1841+591.

We also notice that the faint X-ray source RX J1836.2+5925 that was proposed as a possible counterpart is well within the AGILE error box of AGL J1836+5926. A hint of variability of this source was noticed when comparing two HRI ROSAT observations taken almost three years apart (Mirabal et al. 2001), prompting a claim for the discovery of a new class of compact gamma-ray sources (Mirabal et al. 2000). However, subsequent Chandra X-ray observations showed a practically constant X-ray flux of RX J1836.2+5925, and weakened the variability claim in favour of a scenario encompassing a constant Geminga-like source.
We note here that our group observed the region containing RX J1836.2+5925 with the SWIFT XRT and XMMNewtonduring the period May-June 2008. Analysis of these data and a full discussion of the AGL J1836+5926 counterpart problem will be presented elsewhere.

Future continuous monitoring (both by AGILE and GLAST) is needed to confirm the gamma-ray flux variability and to unveil the source origin.

\section{References}

Barbiellini, G., Fedel, G., Liello, F., et al. 2002, NIM A, 490, 146 Bignami, F. B., \& Caraveo, P. A. 1996, ARA\&A, 34, 331

Caraveo, P. A., Bignami, F. B., \& Trumper, E. T. 1996, A\&ARv, 7(3) Feroci, M., Costa, E., Soffitta, P., et al. 2007, Nucl. Inst. Meth. A, 581, 728 Halpern, J. P., \& Wang, F. Y.-H. 1997, ApJ, 477, 905

Halpern, J. P., Gotthelf, E. V., Mirabal, N., \& Camilo, F. 2002, ApJ, 573, 41 Halpern, J. P., Camilo, F., \& Gotthelf, E. V. 2007, ApJ, 668, 1154 Hartman R. C., Bertsch, D. L., Bloom, S. D., et al. 1999, ApJS, 123, 79 Labanti, C., Argan, A., Bulgarelli, A., et al. 2006, Nucl. Phys. B Proc. Suppl., 150,34

Massaro, E., Giommi, P., Sclavi, S., et al. 2007, AIP Conf. Proc., 921, 349 Mirabal, N., Halpern, J. P., Eracleous, M., \& Becker, R. H. 2000, ApJ, 541, 180 Nolan, P. L., et al. 1994, Proc. 2nd Compton Symposium, AIP Conf. Proc., 304 Nolan, P. L., Bertsch, D. L., Chiang, J., et al. 1996, ApJ, 459, 100 Nolan, P. L., Tompkins, W. F., Grenier, I. A., et al. 2003, ApJ, 597, 615

Mattox, J. R., Bertsch, D. L., Chiang, J., et al. 1996, ApJ, 461, 396

Mattox, J. R., Hartman, R. C., \& Reimer, O. 2001, ApJ, 135, 155

McLaughlin, M. A., Mattox, J. R., Cordes, J. M., \& Thompson, D. J. 1996, ApJ, 473,763

Mirabal, N., \& Halpern, J. P. 2001, ApJ, 547, 137

Perotti, F., Fiorini, M., Incorvaia, S., Mattaini, E., \& Sant'Ambrogio, E. 2006, Nucl. Inst. Meth. A, 556, 228

Prest, M., Barbiellini, G., Bordignon, et al. 2003, Nucl. Inst. Meth. A, 501, 280 Reimer, O., Brazier, K. T. S., Carraminana, A., et al. 2001, MNRAS, 324, 772 Tavani, M., et al. 2008, A\&A, submitted [arXiv:0807.4254v1] Torres, D. F., Romero, G. E., Combi, J. A., et al. 2001, A\&A, 370, 468

Totani, T., Kawasaki, W., \& Kawai, N. 2003, The Proceedings of the IAU 8th Asian-Pacific Regional Meeting, Vol. I, ASP Conf. Proc., 289 